\title{
Diffusion Mechanism of Radiation of a Charged Particle on the Randomly Spaced Dust Grains in the X-rays
}

\section{Zh. S. Gevorkian}

Institute of Radiophysics and Electronics, Ashtarak-2, 378410, Armenia

\author{
V. V. Hambaryan and A. A. Akopian
}

Byurakan Astrophysical Observatory, Byurakan, 378433, Armenia

\section{Diffusion radiation in the $\mathrm{X}$-ray region}

The theory of diffusion radiation of a charged particle on the fluctuations of the dielectric constant developed by Gevorkian can be explained as follows:

A charge moving in a medium creates an electromagnetic field (pseudophoton) which is scattered on the fluctuations of the dielectric constant (here, dust particles) and converted into radiation. In the wavelength region $(l \ll \lambda \ll L)$ ( $l$ is the mean free path of the photon in the medium, $L$ is the characteristic size of the system) the main mechanism of the radiation is the diffusion of the pseudophoton (Gevorkian \& Atayan 1990, Gevorkian 1992, Gevorkian 1993).

For relativistic energies of charged particles $\left(\gamma>10^{3}\right)$ and characteristic sizes of dust particles $\left(a \sim 10^{-5}-10^{-6} \mathrm{~cm}\right)$, the characteristic frequency of diffusion radiation $\left(\omega_{0} \sim \frac{c \gamma}{a}\right)$ lies in the $\mathrm{X}$ - ray region. In this case, the intensity of diffusion radiation of one charged particle is given by (Gevorkian et al. 1998):

$$
I^{D}(\omega)=6 \pi e^{2} \frac{l_{\text {in }}(\omega)}{l^{2}(\omega)}\left(2 \ln \frac{c \gamma}{a \omega}-1\right)
$$

where $l_{\text {in }}$ is the inelastic mean free path:

$$
l_{i n}=\frac{1}{n_{H}\left(\sigma_{H}+\frac{\sum n_{i} \sigma_{i}}{n_{i}}\right)}
$$

where $n_{H}$ is the concentration of $H$ atoms, $\sigma_{H}$ is the photoionization cross section of $H$ atoms , $n_{i}$ and $\sigma_{i}$ are concentration and cross section of the i-th element.

Thus the frequency dependence of $l_{\text {in }}$ and $I^{D}$ is determined by the frequency dependence of the photoeffect cross section. The elastic scattering mean free path is equal to:

$$
l(\omega)=\frac{9 \omega^{2} c^{2}}{4 \pi n a^{4} \omega_{p}^{4}}
$$

where $n$ is the number density of dust particles, $\omega_{p}$ is plasma frequency. 

1998):

The contribution of single scattering radiation is equal to (Gevorkian et al.

$$
I^{0}(\omega) \approx 2 \pi e^{2} \frac{1}{l(\omega)}\left(2 \ln \frac{c \gamma}{a \omega}-1\right) .
$$

Consequently, radiation emitted when charged particles pass through a dusty cloud could be much greater (by order of $l_{i n} / l$ ) if one takes into account the diffusion term of radiation. We have estimated that in the range $2-10 \mathrm{keV}$, $l_{\text {in }} / l$ can be at least $10-100$. In dense dusty clouds $l_{\text {in }} / l$ may reach up to $10^{3}$.

Using photoionization cross sections calculated by Brown \& Gould (1970) we have obtained the theoretical spectrum of diffusion radiation. The spectral index for the range $2-10 \mathrm{keV}$ is equal to -0.8 . In the general case, the expected indices lie in the range from -0.5 to -1.3 (Gevorkian et al. 1998).

\section{AGNs as diffusion $\mathrm{X}$ - ray radiation sources?}

A review of observational data obtained in the range $1-10 \mathrm{keV}$ let us select AGNs as possible sources of $\mathrm{X}$-ray diffusion radiation. The following observational evidence supports our selection:

- Extended X-ray emission (up to $1 K p c$ in size) has been found around the nuclei of AGNs.

- In the nuclei of AGNs are all the necessary conditions (dust and relativistic electrons) for creating diffusion X-ray radiation. Most AGNs are a power source of IR and/or radio emission. There are some correlations between $\mathrm{IR} /$ radio and $\mathrm{X}$-ray radiation. There is also a tight correlation between radio and $\mathrm{X}$-ray morphologies.

- The spectral indices in $2-10 \mathrm{keV}$ of an overwhelming majority of AGNs lie between -0.4 and -1.1 with the mean $\sim-0.8$.

- In the expected range of diffusion radiation $(\sim 1-10 \mathrm{keV})$ observed spectra of AGNs often differ from the spectra of neighboring wavebands.

- The estimates (Gevorkian et al. 1998) of the X-ray luminosities of AGNs in the framework of a simple physical model are consistent with observational data.

\section{References}

Brown, R.L., \& Gould,R.J., 1970, Phys.Rev.,D1, 2252

Gevorkian, Zh.S. 1992, Phys.Lett., A162, 187

Gevorkian, Zh.S. 1993, Radiofizika, 36, 36

Gevorkian, Zh.S. \& Atayan, S.R. 1990, Phys.Lett., A144, 273

Gevorkian, ZH.S.\& Atayan, S.R. 1990, Sov.Phys. JETP 71 (5), 862

Gevorkian, Zh.S., Hambarian,V.V., \& Akopian, A.A., 1998, Astrofizika, 41, 443 